\title{
Measurements of the Proton and Helium Spectra from CREAM-V
}

\author{
T. Mernik ${ }^{* 1}$, Y. Amare ${ }^{1}$, T. Anderson ${ }^{2}$, D. Angelaszek ${ }^{1,3}$, N. Anthony ${ }^{3}$, K. Cheryian $^{3}$, \\ G.H. Choi ${ }^{4}$, M. Copley ${ }^{1}$, S. Coutu ${ }^{2}$, L. Derome ${ }^{5}$, L. Eraud ${ }^{5}$, L. Hagenau ${ }^{3}$, J.H. Han ${ }^{1}$, \\ H.G. Huh ${ }^{1}$, S. Im ${ }^{2}$, J.A. Jeon ${ }^{4}$, S. Jeong ${ }^{4}$, K.C. Kim ${ }^{1}$, M.H. Kim ${ }^{1}$, H.Y. Lee ${ }^{4}$, J. Lee ${ }^{4}$, \\ M.H. Lee ${ }^{1}$, J. Liang ${ }^{3}$, J.T. Link ${ }^{6}$, L. Lu ${ }^{3}$, L. Lutz ${ }^{1}$, A. Menchaca-Rocha ${ }^{7}$, J.W. Mitchell ${ }^{6}$, \\ S.I. Mognet ${ }^{2}$, S. Morton ${ }^{3}$, M. Nester ${ }^{3}$, S. Nutter ${ }^{8}$, O. Ofoha ${ }^{1}$, I.H. Park ${ }^{4}$, N. \\ Picot-Clémente $^{1}$, R. Quinn ${ }^{3}$, E.S. Seo ${ }^{1,3}$, J.R. Smith ${ }^{1}$, P. Walpole ${ }^{1}$, R.P. Weinmann ${ }^{1}$, \\ J.Wu ${ }^{1}$, Y.S. Yoon ${ }^{1}$
}

1 Inst. for Phys. Sci. and Tech., University of Maryland, College Park, MD 20742, USA

2 Dept. of Physics, Penn State University, University Park, PA 16802, USA

3 Dept. of Physics, University of Maryland, College Park, MD 20742, USA

4 Dept. of Physics, Sungkyunkwan University, Suwon 440-746, Republic of Korea

5 Laboratoire de Physique Subatomique et de Cosmologie, UJF - CNRS/IN2P3 - INP, 53 rue des Martyrs, 38026 Grenoble Cedex, France

6 Astroparticle Physics Laboratory, NASA Goddard Space Flight Center, Greenbelt, MD 20771, USA

7 Instituto de Fisica, Universidad Nacional Autonoma de Mexico, Circuito de la Investigación s/n, Ciudad Universitaria, CP 04510 México Distrito Federal, Mexico

8 Dept. of Physics and Geology, Northern Kentucky University, Highland Heights, KY 41099, USA

\begin{abstract}
The Cosmic Rays Energy And Mass (CREAM) balloon payload directly measures the composition and elemental spectra of cosmic rays in the upper stratosphere. It is designed to probe the acceleration mechanism and propagation history of cosmic rays at energies from $10^{12}$ up to $10^{15}$ $\mathrm{eV}$. Being the fifth flight in a series of seven, CREAM-V took data above Antarctica for 39 days from December $1^{\text {st }} 2009$ to January $8^{\text {th }}$ 2010. The instrument comprises a tungsten/scintillating fiber calorimeter using graphite as a target for the energy measurement which had been calibrated at CERN (European Organization for Nuclear Research). The charge measurement of the incident particles is performed by means of a Silicon Charge Detector (SCD), a Cherenkov Detector, a Cherenkov Camera (CherCam) and a Timing Charge Detector (TCD). In this paper we present results from the on-going data analysis and compare them to data collected by the previous CREAM-III flight.
\end{abstract}

35th International Cosmic Ray Conference - ICRC2017

10-20 July, 2017

Bexco, Busan, Korea

\footnotetext{
*Email: tmernik@umd.edu

${ }^{\dagger}$ Speaker.
} 


\section{Introduction}

The CREAM (Cosmic Ray Energy And Mass) Collaboration has conducted a series of stratospheric balloon borne experiments to directly determine the elemental spectra of cosmic rays in the energy range from $10^{12}$ to $10^{15} \mathrm{eV}$. CREAM serves to probe cosmic ray acceleration and galactic propagations mechanisms [1]. The instrument comprises a number of interchangable detectors for charge and energy measurement. Since 2004, seven missions (including the latest named BACCUS - Boron And Carbon Cosmic rays in the Upper Stratosphere) have been launched in Antarctica and taken data on circumpolar flights between a few and several weeks each with slightly varying instrument configurations. In this paper we present preliminary energy and charge measurements - major prerequisites for the proton and helium flux determination - as measured by CREAM-V and compare the findings to the previous CREAM-III mission in particular, due to the identical hardware configuration of both missions .

\section{The CREAM-V Mission}

CREAM-V was launched from McMurdo Station in Antarctica on December $1^{\text {st }} 2009$ and was afloat for 39 days to January $8^{\text {th }} 2010$. The instrument's setup was similar to the previous CREAM-III mission [2]. Centerpiece of the payload was a tungsten/scintillating fiber calorimeter with a graphite target for the energy measurement. It is installed below four charge detectors: Topand Bottom Silicon Charge Detector (SCD), a Cherenkov Detector (CD), a Cherenkov Camera (CherCam) and a Timing Charge Detector (TCD). (Fig.1) Each detector is controlled and read out by designated electronics boards which are connected to a science flight computer (SFC).

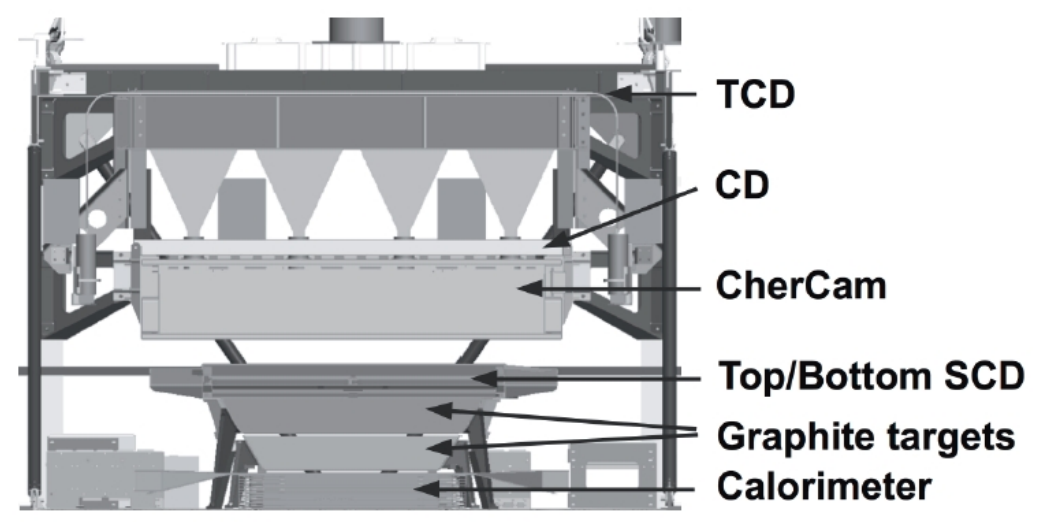

Figure 1: The configuration of the CREAM-V instrument from top to bottom: Timing Charge Detector (TCD), Cherenkov Detector (CD), Cherenkov Camera (CherCam), Top- and Bottom Silicon Charge Detector (T-/B-SCD) and the calorimeter with its graphite targets on top. [3]

The SFC saves all the housekeeping and science data to disk and transmits a part of it via NASA's TDRSS satellites to the Science Operations Center (SOC) on ground for further processing such as real time monitoring of the instrument's health and status. 


\section{Data Analysis}

In this current and preliminary data analysis of the CREAM-V flight we restrict ourselves exclusively to the telemetered data which contains approximately $9 \cdot 10^{5}$ cosmic rays events. Due to a number of longer data link outages during the flight, the actually recorded data set is significantly larger. In the near future we will extend our analysis to the entire data set recovered from the hard drives of the payload which had not been transmitted to ground during operations.

\subsection{Trigger and Quality Cuts}

The events analyzed in the scope of this preliminary analysis have been triggered by the calorimeter using the requirement of six consequetive layers being traversed by the shower track. During our analysis we apply an additional software trigger to select only events for which each of these layers has at least one activated ribbon exceeding the threshold of $15 \mathrm{MeV}$. The conversion of raw adc counts to an energy value in $\mathrm{MeV}$ is conducted by applying the calorimeter calibration, which had been derived from beam line tests (See Sec. 3.4). Among these events we select only those with a trajectory that intersects the active areas of both, Top- and Bottom-SCD as well as the bottom layer of the calorimeter.

\subsection{Track reconstruction}

To determine the trajectory of the cosmic rays penetrating the instrument we compute the axis of the secondary particle shower that is created when the primary cosmic ray hits the graphite target above the calorimeter. Its projection in the $\mathrm{XZ}$ and $\mathrm{YZ}$ planes is reconstructed by a least-squares fit in accordance to earlier CREAM flights [4, 3]. In this way the arrival direction of the incident particle can be estimated. When we analyze the distribution of inclination angles of the arriving

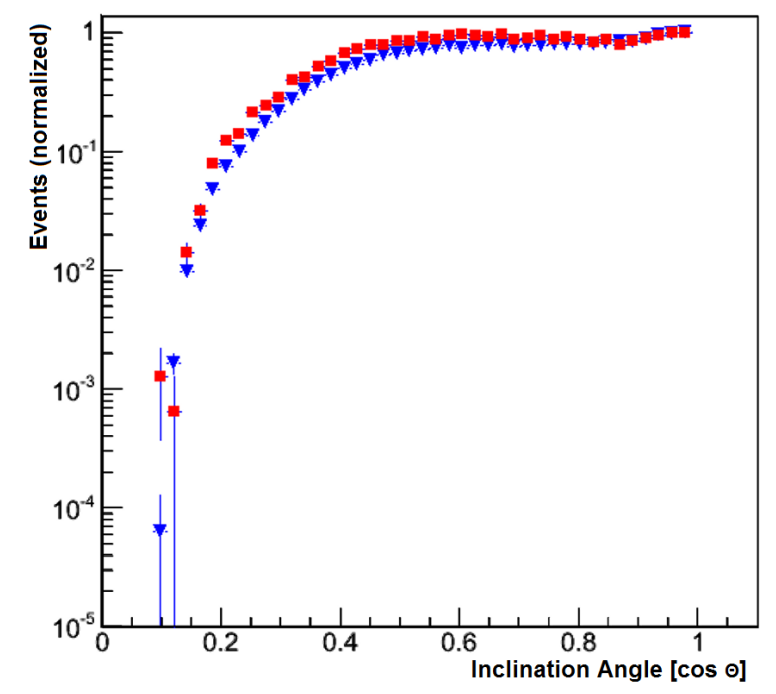

Figure 2: Incident angle inclination [cos $\Theta$ ] distribution of cosmic rays for the CREAM-III mission (blue triangles) and the CREAM-V mission (red squares). The event numbers are normalized to the bin content.

particles we can clearly see the isotropy of cosmic rays. (See Fig. 2) Between $0^{\circ}(\arccos 1)$ and 
$60^{\circ}(\arccos 0.5)$ the events arrive uniformly. The drop above $60^{\circ}$ reflects the geometric acceptance of the detector.

\subsection{Charge Measurement}

In this preliminary study we use the Top- and the Bottom-SCD to determine the charge of the particles traversing the instrument. After the previoulsly described track reconstruction, the SCD pixel which is intersected by the particle's trajectory is scanned together with the surrounding area $(7 \times 7$ pixel). Here we select the pixel with the highest signal counts. The signal corresponds to the ionization energy loss of the incident particle in the silicon layer of the detector. It is approximately proportional to $Z^{2}$. To prevent backscattering particles and electronics noise from contaminating the measurement we consider only those events which feature a similar charge $\left(\Delta Z=\mid Z_{T S C D}-\right.$ $Z_{B S C D} \mid<0.7$ ) in both SCDs. (Fig.3)
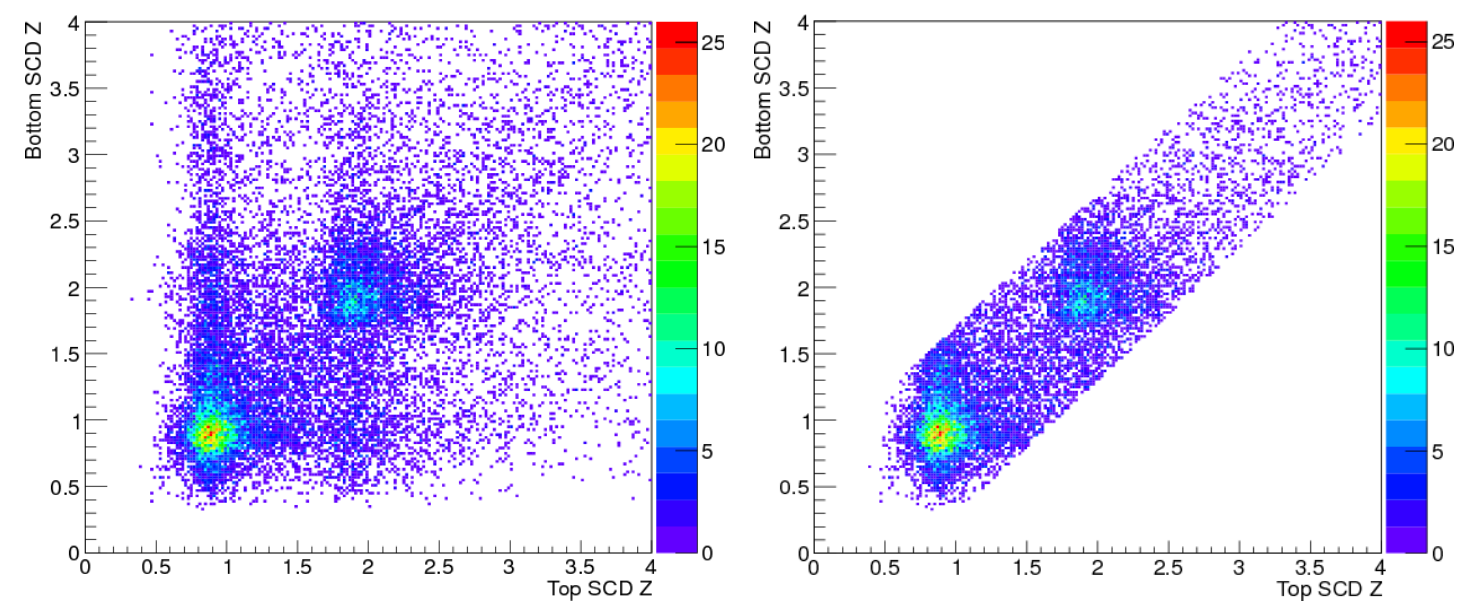

Figure 3: Plotting the charge measured by the Bottom-SCD versus the charge measured by the Top-SCD before (left) and after applying the cut (right). We can see the distributions of proton and helium events clearly in the diagonal of the plot.

\subsection{Energy Spectra}

When the particles strike the carbon target above the calorimeter, hadronic showers are created. The secondary particle's energy is deposited in the layers of tungsten/scintillating fibers of the calorimeter. The sum of the calibrated signals is proportional to the initial energy of the primary particle. The calibration of the detector has been conducted by beam test at CERN (European Organization for Nuclear Research) with a $150 \mathrm{GeV}$ electron beam. The same calorimeter has been utilized for a number of CREAM flights, always showing a remarkably stable performance [5]. All events for this analysis have triggered at least six consecutive layers of the calorimeter, each exceeding the threshold of $15 \mathrm{MeV}$. The events identified as protons in this plot have been selected with a charge $0.5<\mathrm{Z}<1.7$ (for helium $1.7<\mathrm{Z}<2.53$ respectively) as measured by both SCDs. It is important to note that due to the overlapping Landau tails of both, hydrogen and helium peaks we misidentify a certain percentage at this stage of the analysis. Hence, this method can only be regarded as a preliminary approximation. 

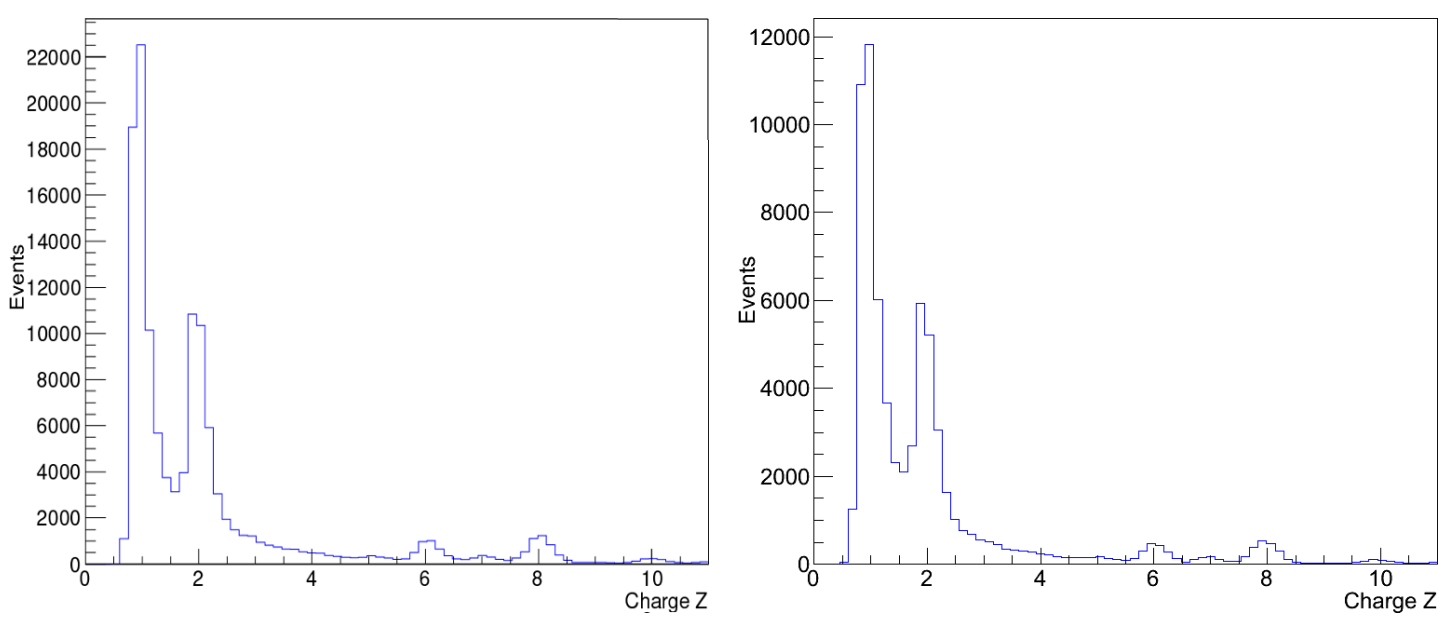

Figure 4: Number of events plotted against the average charge from both SCD detectors. Apart from the prominent $\mathrm{H}$ and He peaks, carbon, nitrogen and oxygen can also clearly be identified. Left: Charge distribution as measured by CREAM-III. Right: CREAM-V.

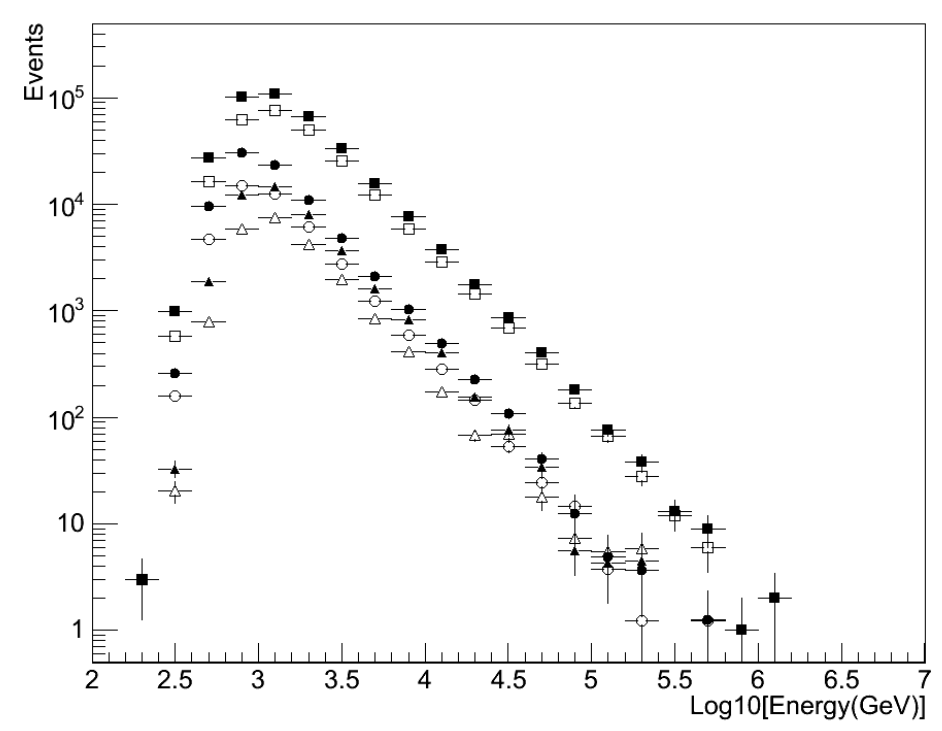

Figure 5: Energy spectra by the CREAM-III (filled symbols) and CREAM-V (open symbols) calorimeters. Additionally to the all particle spectra (squares) we plot the spectra for protons (circles) and helium (triangles). The shape of the spectra follows a power law distribution. In the low energy part of the spectrum the trigger efficiency of the instrument decreases. Thus we observe a drop in the recorded events.

\section{Conclusion}

The two CREAM missions compared in this paper share most major constituents such as the instrument design (both instruments share virtually the same hardware) as well as the duration 
and setting of the flights. In the context of this preliminary CREAM-V data analysis we have exclusively taken the telemetered data into account and therefore neglected a large part of the recorded events. For the analysis of the CREAM-III mission the entire data set was taken into account. The energy spectra recorded by both missions follow the same power law distribution. At this stage of our ongoing analysis we consider this behavior as a good confirmation of the very stable performance of the CREAM instrument. We look forward to apply the same data reconstruction on the full set of the CREAM-V data in the near future.

\section{Acknowledgement}

The authors wish to thank the NASA GSFC WFF for project management and engineering support, and the NASA JSC ISS Program Office for the launch support and the ISS accommodation. This work was supported in the U.S. by NASA grants NNX11AC52G, NNX08AC15G, NNX08AC16G and their predecessor grants, as well as by directed RTOP funds to NASA GSFC WFF. It is supported in Korea by the Creative Research Initiatives of MEST/NRF and by National Research Foundation Grants NRF - 2014R1A2A2A01002734, NRF - 2014R1A1A2006456, NRF 2015R1A2A1A13001843. It is supported in France by IN2P3/CNRS and CNES and in Mexico by DGAPA-UNAM and CONACYT. The authors also thank H.S. Choi, Korea Institute of Industrial Technology, for contributions to the SCD thermal vacuum tests, M. Geske, Penn State, for contributions to the BSD, and M. A. Coplan, University of Maryland, contributions to CAL electronics vacuum tests.

\section{References}

[1] Ahn, H.S., et al.: The Cosmic Ray Energetics And Mass (CREAM) instrument, Nuclear Instruments and Methods in Physics Research Section A, Vol. 579, 1034, 2007

[2] Yoon, Y.S, et al.: Preliminary Proton and Helium Spectra from the CREAM-III Flight, Proceedings of the 31st ICRC, Lodz, 2009

[3] Yoon, Y.S, et al.: Proton and Helium Spectra from the CREAM-III flight, The Astrophysical Journal, Vol. 839, No.1, 2017

[4] Ahn, H. S., et al.: Cosmic-Ray Proton and Helium Spectra from the First CREAM Flight, The Astrophysical Journal, Vol. 728, No.2, 2011

[5] Han, J.H., et al.: Performance of the CREAM-V and CREAM-VI calorimeters in flight, Proceedings of the 32nd ICRC, Beijing, 2011 